\title{
Object-Based Control of Attention Is Sensitive to Recent Experience
}

\author{
Hyunkyu Lee \\ University of Illinois
}

\author{
Arthur F. Kramer \\ University of Illinois
}

\author{
Michael C. Mozer \\ University of Colorado \\ Shaun P. Vecera \\ University of Iowa
}

\begin{abstract}
How is attention guided by past experience? In visual search, numerous studies have shown that recent trials influence responses to the current trial. Repeating features such as color, shape, or location of a target facilitates performance. Here we examine whether recent experience also modulates a more abstract dimension of attentional control, object-based and location-based control. Participants performed a cued target discrimination task with stimuli presented on 2 rectangles. Response times to targets appearing in an uncued location on a cued rectangle were faster than to targets on the uncued rectangle, demonstrating an object-based attentional benefit. We investigated the object-based benefit on the current trial contingent on the cue-target relationship on the previous trial. The object-based benefit was significant only when the cued object contained the target on the previous trial, not when the uncued object contained the target. This effect of recent experience was not due to either the repetition of spatial cue-target location or the repetition of the response, but to adaptation to contingencies in the environment. Our results suggest a unifying view of attentional control that spans the concrete dimensions of control (e.g., determining the relative importance of red vs. blue) to the abstract (determining the relative importance of objects vs. locations in space). Attention closely tracks the short time scale structure of the environment and automatically adapts to optimize performance to this structure.
\end{abstract}

Keywords: object-based attention, control setting, recent experience, adaptation

Picture yourself at a busy train terminal searching for a friend. At any given moment, your visual system is confronted with a massive quantity of information. To find your friend in a bustling and constantly moving crowd, you have to filter the spatiotemporal stream into a manageable quantity by granting priority to task-relevant items (i.e., your friend and others that look like her) and locations (i.e., where in the visual field people are likely to be standing). Directing attention to your friend involves attentional control, the guidance of attention in a task and goal relevant manner.

Successful guidance of attention may involve following instructions, for example, if your friend has advised you to look for her yellow hat or that she is standing near the top of the stairs. More generally, however, attentional control depends on past experience-experience with your friend's appearance, and with the broader population's appearance, which is key to directing your search based on features that are likely to discriminate your friend from all the other people in the station. If her hair color or height is distinctive, those features can guide attention.

This article was published Online First October 3, 2011.

Hyunkyu Lee and Arthur F. Kramer, Department of Psychology and Beckman Institute, University of Illinois; Michael C. Mozer, Department of Computer Science and Institute of Cognitive Science, University of Colorado; Shaun P. Vecera, Department of Psychology, University of Iowa.

Correspondence concerning this article should be addressed to Hyunkyu Lee, Beckman Institute, 405 North Mathews Avenue, Urbana, IL 61802. E-mail: hyunklee@illinois.edu

\section{Control Settings}

Attentional control can be cast in terms of control settings, which specify modulations of the operation of visual attention in the service of performing a task or achieving a goal (Folk \& Remington, 1998; Folk, Remington, \& Johnson, 1992; Folk, Remington, \& Wright, 1994). The guided search model (Wolfe, 1994, 2007) suggests a mechanism for adjusting control settings to direct attention to locations containing target-related visual features. For example, if your friend has a yellow hat or blonde hair, the attentional weight or gain can be turned up on primitive features encoding the color yellow to bias selection in favor of locations with yellow objects.

Beyond top-down modulation of feature gains, the attention literature also has delineated other forms of attentional control. For example, there is empirical support for a distinction between directing attention to a spatial location (location-based attentional control) and directing attention to an object (object-based attentional control). Although there is still a debate on the mechanism of object-based control-whether attention spreads automatically within the boundaries of objects (Davis, Driver, Pavani, \& Shepherd, 2000; McCarley, Kramer, \& Peterson, 2002; Richard, Lee, \& Vecera, 2008) or whether objects merely affect the attention strategy by prioritizing locations within an attended object (Moore, Yantis, \& Vaughan, 1998; Avrahami, 1999; Shomstein \& Yantis, 2002) - it is well agreed that interactions are possible between location- and object-based attentional control (Müller \& Kleinschmidt, 2003; Soto \& Blanco, 2004; Vecera, 1994). These two forms of control appear to coexist and operate in different situations (e.g., Vecera \& Farah, 1994) or simultaneously 
within an experiment (e.g., Egly, Driver, \& Rafal, 1994; Kramer \& Jacboson, 1991; Vecera, 1994), depending on the specific paradigm used.

In this article, we explore whether an attentional control setting might exist to arbitrate between location-based and object-based guidance of attention based on task demands. Moreover, because this hypothetical setting would need to be determined for each task, we investigate the issue of how control is established. It is possible that a task description is sufficient to determine the appropriate setting, and it will remain fixed and stable over the course of an experiment as the task is repeatedly performed. The notion that explicit instructions allow an individual to configure attention to a task underlies many theories of top-down control (e.g., Deismone \& Duncan, 1995; Folk et al., 1992). However, it is also possible that experience with a task is required to adapt the control settings for that task (e.g., Cosman \& Vecera, 2010; Neo \& Chua, 2006). This experience may be on a long time scale-over the course of a block of trials based on block-wide item probabilities (e.g., Neo \& Chua, 2006; Geyer, Müller, \& Krummenacher, 2008) — or it might be on a much shorter time scale_tuned from trial to trial based on recent experience (Cosman \& Vecera, 2010; Pinto, Olivers, \& Theeuwes, 2005).

We return shortly to the issue of object- versus locationbased control of attention, but first we discuss the literature addressing the nature of experience required to adapt attentional control.

\section{Adaptation of Attentional Control}

Evidence for long time-scale adaptation comes from studies that examine learning over the course of dozens of trials about regular patterns in the stimulus environment (e.g., Chun \& Jiang, 1998, contextual cueing). Evidence for short time-scale adaptation is obtained by examining the influence of the trial-by-trial repetition of features on the speed and accuracy of responding. When features of a target or distractors such as color, shape, location, orientation, or size are repeated from one trial to the next, performance is facilitated (e.g., Maljkovic \& Nakayama, 1994, 1996, 2000). This type of priming also has been demonstrated not only for specific features but also for entire feature dimensions (e.g., color vs. shape, dimension repetition benefit; Found \& Müller, 1996; Mortier, Theeuwes, \& Starreveld, 2005) and for higher order properties (e.g., Lamy, Amunts, \& Bar-Haim, 2008). These trialto-trial adjustments in performance are readily interpreted in terms of adaptation of attentional control (see Kristjánsson \& Campana, 2010, for a recent review).

In a series of studies, Maljkovic and Nakayama (1994, 1996, 2000; also see Hillstrom, 2000; Huang, Holcombe, \& Pashler, 2004) demonstrated a short-lived, implicit memory influence on a visual search task. On each trial, a search display was presented that contained three notched diamonds. One diamond had a distinct color from the other two diamonds, defining a singleton target (either a green diamond among red or a red diamond among green). Participants reported whether the singleton target had a notch on its left or right side. Because the target was defined by a single feature, it was detected rapidly regardless of the number of items in a display. Even though search was efficient and the target popped out, the results showed a clear benefit from the repetition of the defining feature of the target. Response times decreased significantly when the color singleton was carried over from trial to trial, and this benefit extended to the half dozen trials leading up to the current trial.

Learning of attentional control from previous experience can be found not only from the repetition of a simple visual feature but also from the repetition of an abstract spatial relationship (Kristjánsson, Mackeben, \& Nakayama, 2001; see also Becker, 2008; Kristjánsson \& Nakayama, 2003; Sigurdardottir, Kristjánsson, \& Driver, 2008). Kristjánsson and colleagues (2001) investigated whether the allocation of attention can be modulated by a consistent relationship between a cue (two parallel horizontal lines) and a target (an eye-shaped object lying between the two cue lines). The absolute position of the target in the display varied randomly from trial to trial. In addition, the relative position of the target with respect to the cue varied: The target could appear on either the left or right end of the cue. In a critical "streak" condition, there were more repetitions than changes of the relative target position with respect to the cue. In other words, in the streak condition, there would be a sequence of trials in which the target appears on the same side of the cue. The streak condition performance was contrasted with performance under a "switch" condition where the cue-target relationship was completely predictable (e.g., trial $n-1$ target on the left side of the cue, trial $n$ target on the right side of the cue). Results showed a significantly higher accuracy in the streak condition than in the switch condition, suggesting that short-term learning of the cue-target relationship took place.

The mechanisms underlying these intertrial priming effects are still a matter for debate (e.g., Kristjánsson \& Campana, 2010; Meeter \& Olivers, 2006, for a review). One view holds that repeating the target feature produces early facilitation by adapting attentional control settings-for example, the gains along various feature dimensions (Becker, 2008; Chun \& Nakayama, 2000; Lee, Mozer, \& Vecera, 2009; Mozer \& Baldwin, 2008; Sigurdardottir et al., 2008). Another view suggests that priming affects a late, postperceptual stage such as response selection (Huang et al., 2004; Huang \& Pashler, 2005). For example, Huang and Pashler (2005) found only a small priming effect of orientation repetition during feature search of brief displays (see also Becker, 2008; Sigurdardottir et al., 2008, for counterexamples). Recently, several studies suggested a dual-stage account of intertrial priming that involves both an early perceptual/attentional stage and a later, response-relate stage of visual search (Lamy, Yashar, \& Ruderman, 2010; Töllner, Gramann, Müller, Kiss, \& Eimer, 2008; Töllner, Zehetleitner, Gramann, \& Müller, 2010). Using event-related potentials, Töllner and colleagues (2008, 2010) demonstrated that feature repetition enhanced amplitudes of the posterior contralateral negativity ( $\mathrm{PCN})$, suggesting a facilitated allocation of attentional resources to the target, whereas the repetition of response influenced the lateralized readiness potential (LRP), suggesting a facilitation in response selection.

In summary, although intertrial priming effects can be obtained at a postperceptual stage of response selection, they also occur at the stage of attentional control. Trial-to-trial influences on attentional control range from the concrete (e.g., determining the weighting of feature dimensions) to the abstract (e.g., determining the relationship between cue and target). 


\section{Object-Based Attention}

The control of attention by both spatial and object constraints in a single task is well established and is best demonstrated by the cued spatial detection task developed by Egly et al. (1994, our version of which is depicted in Figure 1. In their study, Egly et al. had participants view two rectangles, followed by a spatial cue that appeared at the end of one of the rectangles (see Figure 1). An onset target followed the cue, and participants were to make a speeded response to the target. The target occurred most frequently at the validly cued location (75\% of trials) and occasionally at an invalidly cued location ( $25 \%$ of trials). Participants detected validly cued targets faster than invalidly cued targets, demonstrating a spatial cueing effect. Beyond this basic spatial cueing effect, however, there were two types of invalidly cued targets: Those appearing at the uncued end of the cued object (invalid-same) and those appearing at the uncued object (invalid-different). Because both of these invalid trial types were equidistant from the cued location, spatial attention alone would predict no difference in performance. However, invalid-same targets were detected faster than invalid-different targets, demonstrating an object-based component to the control of attention.

Object-based attentional effects occur under many conditions. For example, object-based attention operates over perceptually completed objects (e.g., Moore et al., 1998; Pratt \& Sekuler, 2001), suggesting that perceptual completion processes operate before attention is applied to objects. Object-based effects are also observed across a variety of tasks; Egly et al. (1994) used target detection, but object-based effects also emerge in target discrimination (Kramer \& Jacobson, 1991; Hecht \& Vecera, 2007; Moore et al., 1998; Watson \& Kramer, 1999; Zemel, Behrmann, Mozer, \& Bavelier, 2002). Finally, both location-based and object-based effects in the Egly et al. task are influenced by experiment-wide cue-target contingencies or probabilities. Two studies are particu-

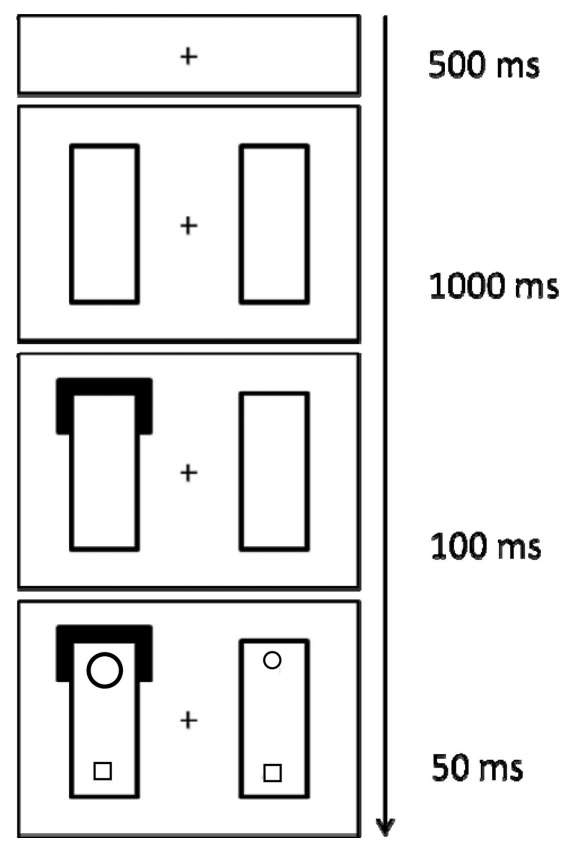

Figure 1. Stimuli and order of events in experiment. larly relevant for understanding how cue predictability influences object-based attention.

He, Fan, Zhou, and Chen (2004) manipulated cue probability and examined the magnitude of location-based and object-based attentional effects. In one experiment, spatially valid cues appeared frequently (66\% of trials) and the two types of invalid cues appeared equally infrequently (10\% of trials in both the invalidsame object and invalid-different conditions, $14 \%$ of trials were catch trials). In another experiment, the cue's spatial validity was reduced (50\% of trials) with a corresponding increase in the frequency of the two invalid cue types (18\% of trials for invalidsame and invalid-different conditions, $14 \%$ of trials were catch trials). He et al. found that location-based effects decreased as the spatial predictability decreased from $66 \%$ to $50 \%$. However, object-based effects persisted as the object predictability, which is the probability that a cue indicates the target appearing object, decreased from $76 \%$ to $68 \%$.

Shomstein and Yantis (2004) used a different probability manipulation that pitted object grouping against the spatial relationship between a cue and target. Participants were cued to a particular location; although most targets appeared at this location (50\%), invalidly cued targets were more likely to appear at one location ( $\sim 42 \%$ of trials) than at another location ( $\sim 8 \%$ of trials). Object structure was orthogonal to this probability manipulation, so that the high and low probability locations were equally likely to appear within the same object or different object as the spatial cue. The Stimulus-Onset Asynchrony (SOA) from cue to target varied (200, 400, or $600 \mathrm{~ms}$ ), and for all SOAs, probabilities affected response times: Invalidly cued targets were identified more rapidly when they appeared in the high-probability location than when they appeared in the low-probability location. It is interesting that object-based effects were present only at the shortest SOA $(200 \mathrm{~ms})$. At the longest SOA $(600 \mathrm{~ms})$, only probability influenced attentional priority. Shomstein and Yantis (2004) concluded that two mechanisms affected the relative strength of object-based and learning-based attentional effects. The first mechanism was a fast acting, mandatory "configural" mechanism based on object grouping (i.e., the structure of the rectangles). A second mechanism was a context-dependent mechanism based on learning. Thus, at the shortest SOA, configural factors contributed to attentional control independent to the contextual factors, whereas at longer SOA, contextual factors could override the configural mechanism; the default object-based effect disappears when the SOAs were long enough to redirect attention based on other information that was a lot more useful (see also Chen \& Cave, 2008, demonstrating no contextual effect on object-based attention).

In terms of the influence of previous experience on object-based attention, He et al. (2004) and Shomstein and Yantis (2004) demonstrated that object-based attention might be independent of regularities in a block of trial-the cue-target probabilities in He et al., and target location probability in Shomstein and Yantis (2004) at least at short SOAs. However, these studies focused on longer term experience by averaging performance over trials within a block. Consequently, these studies did not explore the possibility that object-based attentional control operates on a much shorter term scale. Similar to studies showing that physical features of a target such as color on one trial can modulate the allocation of 
attention on the next trial, it is possible that sequential effects arise in the allocation of object-based attention.

\section{Experiment 1}

In the present experiments, we directly tested whether objectbased attentional effects can be influenced by recent trial history. We were specifically interested in whether the relationship between the cue and target on the previous trial influences the allocation of attention on the current trial. To examine this question, we used Egly et al.'s (1994) task and classified the previous trial's cue-target relationship based on the cued object. If the target appeared on the cued object, we considered that a valid-object cue. If the target appeared on the uncued object, we considered that an invalid-object cue.

We made two modifications to the original Egly et al. (1994) task. First, in the original experiment, $75 \%$ of trials were valid, $12.5 \%$ invalid same, and $12.5 \%$ invalid different. We instead used a $1 / 3$ probability for each trial type to increase the number of invalid trials. Using the original probability of invalid trials leaves only few trials for each condition when analyzed by previous object-cue validity and current-trial's cue-target validity types. Second, instead of $400 \mathrm{~ms}$, we used $100 \mathrm{~ms}$ cue-to-target SOA. The modified probability and cue-to-target SOA does not affect general space-based and object-based cueing effects (see Lee \& Vecera, 2005, 2010, using the same validity probability and SOA). Finally, as we were interested in the trial-to-trial effect, we used a discrimination task that does not require catch trials. A target and three distractors were presented at the four ends of rectangles, and the target was determined by its distinct size. The target was larger than the distractors. Participants were instructed to detect a target and to respond the shape of the target, either a circle or a square, by pressing a corresponding key.

If object-based attentional control settings are stable and show little trial-to-trial modulation, the previous trial's object-cue validity will have a negligible effect on the current trial. In contrast, if object-based attention control can be guided by previous cue-target object validity, there will be modulation of object-based attentional effects on the current trial from the previous trial's object-cue validity.

\section{Method}

Participants. Twenty University of Iowa undergraduates (range 18-30 years) with normal or corrected-to-normal vision volunteered for course credit. Participants signed an informed consent approved by the University of Iowa Institutional Review Board before they participated in the study.

Stimuli. Displays contained two outlined rectangles measuring $1.4^{\circ}$ by $8.8^{\circ}$ of visual angle from a viewing distance of $60 \mathrm{~cm}$. The rectangles were aligned either horizontally or vertically; the far edge of each rectangle was $4.4^{\circ}$ from the fixation cross (see Figure 1). A peripheral cue highlighted the end of one rectangle with a $0.1^{\circ}$ thick line. The cue appeared on each end of rectangles equally often. Next, four stimuli-three small circles or squares $\left(0.4^{\circ}\right.$ in diameter $)$ and one large circle or square $\left(0.9^{\circ}\right.$ in diameter $)$ were presented, with one shape centered at each of the four ends of the rectangles. The target was the large stimulus, and each shape occurred equally often. There was an equal probability that the target appeared on each end of rectangles.
Procedure. Trials began with a fixation cross for $500 \mathrm{~ms}$, followed by two rectangles (1,000 ms). A cue appeared for $100 \mathrm{~ms}$. Following the cue, a target and three distractors were presented (50 $\mathrm{ms}$ ). Observers pressed the $n$ key for a circle target and the $m$ key for a square target. Feedback for incorrect responses was presented for $500 \mathrm{~ms}$ ("wrong").

There were three cue-target relations (current-trial validity conditions: valid, invalid-same, and invalid-different). In the valid condition, a target appeared at the cued location. In the invalidsame condition, a target appeared on the uncued end of the cued object. In the invalid-different condition, a target appeared on the uncued object (at the same distance from the cued location as the invalid-same condition). Those three conditions were presented equally often. There were two previous object-cue validity conditions (valid-object cue and invalid-object cue). Previous valid and invalid-same trials were grouped as valid-object cue trials and previous invalid-different trials were considered as invalid-object cue trials. Participants performed a total of 404 trials and received breaks every 96 trials. The first 20 trials of the experiment were considered as practice and were not analyzed.

\section{Results and Discussion}

Figure 2 shows the reaction time (RT) results. Only trials in which responses to the target discrimination task were correct $(89 \%)$ were analyzed. RTs less than $150 \mathrm{~ms}$ and greater than 2,000 ms were not included in the analysis, and this trimming eliminated less than $1 \%$ of the data. Mean RTs were analyzed with withinsubject analysis of variance (ANOVA), with current-trial validity (valid, invalid-same, and invalid-different) and previous objectcue validity (valid-object cue and invalid-object cue) as factors. There was a significant main effect of current-trial validity, $F(2$, $38)=45.93, p<.01$. The valid trials showed the fastest RTs (562 $\mathrm{ms})$, followed by invalid-same trials $(620 \mathrm{~ms})$. The RTs for invalid-difference trials were the slowest $(640 \mathrm{~ms})$. Pairwise comparisons with Bonferroni adjustment demonstrated that there was location-based effect suggested by significant difference between valid and invalid-same condition, $p<.01$. The difference between the invalid-same and invalid-different was also significant, indicating an object-based effect, $p<.01$. The main effect of previous object-cue validity was not significant, $F(1,19)<1$, ns.

The interaction between current-trial validity and previous object-cue validity was marginally significant, $F(2,38)=2.56$, $p=.09$. Pairwise comparisons revealed that in both the previous valid-object and the invalid-object cue conditions, the difference between valid and invalid-same conditions (i.e., evidence for location-based attention) was significant, $t(19)=6.24, p<.01$, $t(19)=3.92, p<.01$ for previous valid- and invalid-object cue conditions, respectively. However, the difference between invalidsame and invalid-different conditions (i.e., evidence for objectbased attention) was significant only in the previous valid-object cue condition, $t(19)=5.32, p<.01$. In the previous invalid-object cue condition, there was no object-based attention control found, $t(19)=1.08, p>.1$.

Accuracy data were analyzed in the same way as the RT data (see Table 1 for accuracy data). The main effect of current-trial validity was significant, $F(2,38)=6.31, p<.01$. Valid trials $(92.7 \%)$ showed higher accuracy than invalid-same trials $(88.5 \%)$, $t(19)=1.82, p=.085$. Invalid-same trials showed higher 


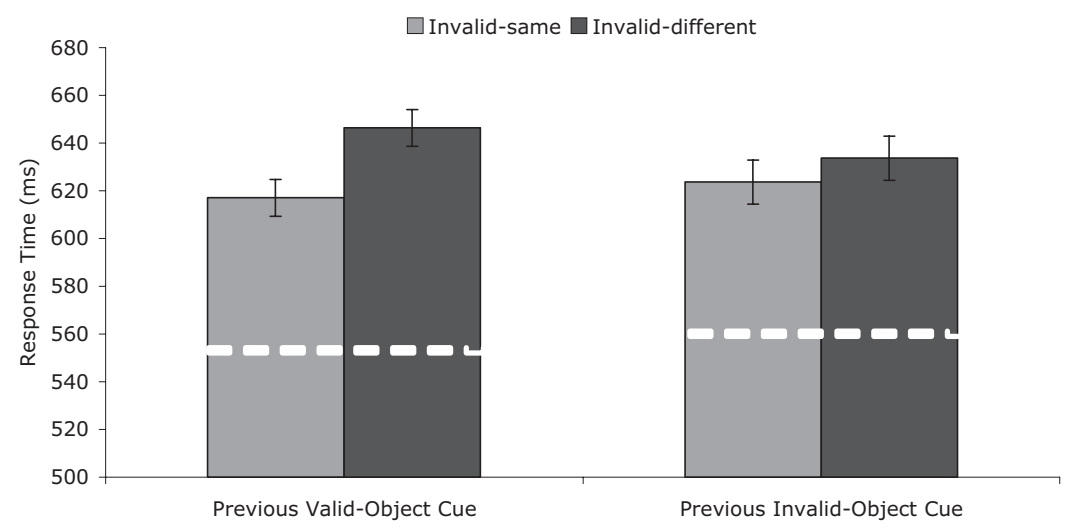

Figure 2. The results of Experiment 1 by previous object-cue validity and current-trial validity condition. Object-based attention effect was found only in the previous valid-object cue condition. The horizontal dashed line represents the valid-cue condition. Error bars are within-subject $95 \%$ confidence intervals.

accuracy than invalid-different trials $(85.7 \%), t(19)=2.99, p<$ .05. Neither the main effect of previous object-cue validity condition, $F(1,19)<1$, ns, nor the interaction was significant, $F(2,38)<1, n s$.

One might argue that the previous object-cue validity is potentially contaminated by spatial attention effects because it combines data from the previous trial regardless of whether the cue was spatially valid. To test the presence of "pure" object-based priming, we performed an analysis in which we considered only data in which the cue was spatially invalid. Thus, we tested an interaction between the current-trial validity (invalid-same vs. invaliddifferent) and the previous-trial validity (invalid-same vs. invaliddifferent). The interaction was significant, $F(1,19)=4.2, p=.05$, indicating that a cue to the correct object but the wrong location primes the deployment of object-based attention. Accuracy data showed no significant interaction, $F(1,19)=.005, p>.05$.

In a similar fashion, we can evaluate pure space-based priming apart from object-based effects by examining the interaction between current-trial validity (valid vs. invalid-same) and the previous-trial validity (valid vs. invalid-same). This interaction is also reliable, $F(1,19)=10.09, p<.01$, indicating that a cue to the target location primes the deployment of space-based attention, but a cue to a nontarget location within the same object does not.
Accuracy data showed no significant interaction, $F(1,19)=1.55$, $p>.05$.

Many experiments in the literature have demonstrated robust priming from the physical repetition of cue or target features. The present experiment extends these results by demonstrating priming of object-cue validity: If on one trial a cue predicts the object on which a target will appear, then the cue is treated as object predictive on the next trial. That is, attentional control processes respond to the cue by directing attention to the cued object. However, when the cue on one trial does not predict the object on which a target will appear, then on the next trial, attention is not preferentially directed to the cued object. These results suggest that guiding attention to an object is modulated by recent experience. If the target appeared on the cued object in the previous trial, the experience enhances object-based attention. In contrast, if the target did not appear on the cued object in the previous trial, the experience eliminated object-based attention.

It is important to note that the priming effect is based on the abstract property of whether the cue and target appear on the same object, not on physical repetition of the cue, target, or object locations. The orientation of display, cue location, and target location were randomly selected for each trial. Therefore, there was very little chance (one in 24) that two identical trials were

Table 1

Accuracy Results of Experiments 1 to 3

\begin{tabular}{|c|c|c|c|c|c|c|c|c|}
\hline & \multicolumn{4}{|c|}{ Previous valid-object cue } & \multicolumn{4}{|c|}{ Previous invalid-object cue } \\
\hline & Valid & Invalid-same & Invalid-near & $\begin{array}{c}\text { Invalid-far ACC } \\
\text { and RT }\end{array}$ & Valid & Invalid-same & Invalid-near & $\begin{array}{c}\text { Invalid-far ACC } \\
\text { and RT }\end{array}$ \\
\hline Experiment 1 & $93(6)$ & $88(11)$ & $85(11)$ & & $92(6)$ & $89(12)$ & $86(12)$ & \\
\hline Experiment 2 & $91(9)$ & $90(11)$ & $89(13)$ & $\begin{array}{c}87(15) \\
643(161)\end{array}$ & $91(9)$ & $89(12)$ & $88(16)$ & $\begin{array}{c}89(14) \\
663(174)\end{array}$ \\
\hline Experiment 3 (SOA 100) & $85(23)$ & $82(24)$ & $89(8)$ & $\begin{array}{c}90(24) \\
567(105)\end{array}$ & $94(8)$ & $89(13)$ & $90(7)$ & $\begin{array}{r}89(15) \\
555(93)\end{array}$ \\
\hline Experiment 3 (SOA 600) & $92(17)$ & $93(14)$ & $91(12)$ & $\begin{array}{r}92(15) \\
574(98)\end{array}$ & $95(4)$ & $93(9)$ & $90(12)$ & $\begin{array}{r}91(10) \\
579(87)\end{array}$ \\
\hline
\end{tabular}

Note. Standard deviations are in parentheses. $\mathrm{ACC}=$ accuracy data; $\mathrm{RT}=$ reaction time; $\mathrm{SOA}=$ Stimulus-Onset Asynchrony. 
repeated. To test the possible influence of perceptual priming on the current results, we conducted a within-subject analysis with two levels of orientation change condition (no-change if two successive trials had identical orientation and change if two successive trials had different orientations), two levels of current-trial validity (invalid-same and invalid-different) and two levels of previous object-cue validity (valid-object cue and invalid-object cue) as factors. If perceptual priming played a role in the effect we observe, there should be a larger priming effect when sequential trials had identical orientations. The results showed that there was no significant main effect of orientation change condition, $F(1$, $19)<1$, ns. It is most important to note that none of the interactions with the orientation change condition were significant, $F(1$, $19)<1, n s ; F(1,19)=1.77, p>.05 ; F(1,19)<1$, $n s$; the interaction between orientation change and current-trial validity, orientation change and previous object-cue validity, and orientation change, current-trial validity and previous object-cue validity, respectively. These results confirm that priming of object-based attentional allocation observed in the present study occurs at an abstract level, not from the repetition of an identical display or cue-target location.

However, in Experiment 1, the number of valid-object trials was twice the number of invalid-object trials. Therefore, it is possible that the failure to find an object-based allocation of attention on the trials following invalid-object trial might not be because of the modulation of attentional control from previous experience but because of the lack of statistical power caused from fewer observations. Further, the finding of an object-based attentional allocation on the trials following valid object trials may be occurring only because of the statistics of cue validity: On 2/3 of the trials in Experiment 1, the cue predicted the object on which the target would appear. Perhaps the object-based effect that we observed occurred not solely because of the previous trial, but because within a block of trials, the cue tended to be a valid object predictor.

\section{Experiment 2}

To rule out these explanations, we conducted an experiment in which the target could appear on any of the four ends of the two objects with equal probability. Thus, we added to the three conditions of Experiment 1 a fourth condition in which the target appeared at the far end of the uncued object, that is, diagonally across from the cued location. To simplify the terminology, we name the conditions in which the target appears on the near and far locations of the uncued object as invalid-near and invalid-far, respectively. Because the cue and target could appear on the four ends of objects equally often, the number of trials following the valid-object cue condition (valid and invalid-same object) and invalid-object cue condition (invalid-near and invalid-far) was the same. Further, because the four trial types occurred with equal frequency, the cue provided no systematic indication of the object on which the target would appear. In addition to changing the number of locations on which the target could occur in Experiment 2 , we also counterbalanced the sequence of the orientation of displays on trial $n$ and trial $n-1$, the cue-target relation of trial $n$ and trial $n-1$, and whether the target on trial $n$ and $n-1$ are in the same location (1/4 trials are same location, 3/4 are different).

We expected that the addition of fourth validity condition (invalidfar) and the equal cue-target probability might interfere with the general cueing effect. The main interest in this experiment, however, was whether the priming of object-based attentional control observed in Experiment 1 would also be found when overall there was no systematic contingency between cue and target.

\section{Method}

Participants. Twenty-two participants (age range 18-24 years) from the Urbana-Champaign, Illinois community were recruited and paid to participate in a 1-hr session. They all had normal or corrected-to-normal visual acuity tested by Snellen Eye chart and normal color vision tested by Ishihara Test Chart Book for color deficiency. Participants signed an informed consent approved by the University of Illinois Institutional Review Board before they participated in the study.

Stimuli and procedure. Stimuli and procedure were identical to those in Experiment 1 with several exceptions. First, there were four validity conditions (valid, invalid-same, invalid-near, and invalid-far). Those four validity conditions were presented equally often. Valid and invalid-same trials were considered as validobject cue condition and invalid-near and invalid-far trials were considered as invalid-object cue condition. The sequences of orientation, cue-target relation, and the target location were counterbalanced throughout the experiment. Participants performed 276 trials and received breaks every 64 trials. The first 20 trials were considered as practice and excluded from the data analysis.

\section{Results and Discussion}

Figure 3 shows the RT results. Only trials in which responses to the target discrimination task were correct $(89 \%)$ were analyzed. RTs less than $150 \mathrm{~ms}$ and greater than 2,000 ms were not included in the analysis, and this trimming eliminated less than $1 \%$ of the data. Because we are only interested in the space-based (valid vs. invalid-same) and object-based (invalid-same vs. invalid-near) attention controls, current invalid-far condition was not included in the analysis (see Table 1 for invalid-far RT). A two-way ANOVA was conducted with current-validity (valid, invalid-same and invalid-near) and previous object-cue validity (valid-object cue and invalid-object cue) as within-subject factors. The main effect of the current-trial validity effect was significant, $F(2,42)=4.97$, $p<.05$. The valid trials showed the fastest RTs $(607 \mathrm{~ms})$, followed by invalid-same $(630 \mathrm{~ms})$, and invalid-near $(643 \mathrm{~ms})$ conditions. Pairwise comparisons with Bonferroni adjustment demonstrated no significant difference between the valid and invalid-same and between invalid-same and invalid-near conditions, indicating no overall space- and object-based attention control. The difference between valid and invalid-near was significant, $p<.01$. The main effect of previous object-cue validity was not significant, $F(1$, 21) $<1$, ns.

It is most important to note that the interaction between currentvalidity and previous object-cue validity was significant, $F(2$, $42)=3.32, p<.05$. Pairwise comparisons revealed that the difference between valid and invalid-same conditions (i.e., evidence for space-based attention) was significant only in the previous valid-object cue condition, $t(21)=2.7, p<.05$. Although there was a trend, the difference between valid and invalid-same trials did not reach significant level in the previous invalid-object cue condition, $t(21)=1.36, p>.05$. Similarly, the difference 


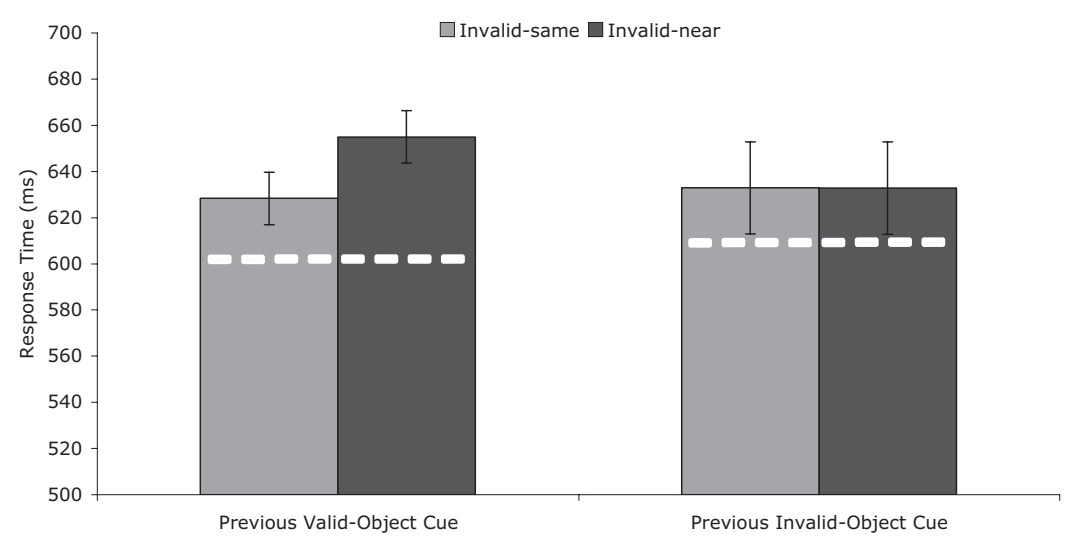

Figure 3. The results of Experiment 2 by previous object-cue validity and current-trial validity condition. Object-based attention effect was found only in the previous valid-object cue condition. The horizontal dashed line represents the valid-cue condition. Error bars are within-subject $95 \%$ confidence intervals.

between invalid-same and invalid-near conditions (i.e., evidence for object-based attention) was significant only in the previous valid-object cue condition, $t(21)=2.21, p>.05$. In the previous invalid-object cue condition, no object-based attention effect was found, $t(21)<1, n s$.

Accuracy data were analyzed with within-subject ANOVA, with current-trial validity (valid, invalid-same, and invalid-near) and previous object-cue validity as factors. Neither main effects nor an interaction was significant, $F(2,42)=1.10, p>.05 ; F(1,21)=$ $2.89, p>.05 ; F(2,42)=.209, p>.05$; for main effect of current-trial validity, previous object-cue validity conditions and the interaction between those two conditions, respectively.

In Experiment 1, we tested pure object-based and pure spacebased priming effects and found them to be reliable. In Experiment 2 , these effects were not reliable. Although there was a trend, the pure object-based priming was not significant, $F(1,21)=1.09$. $n$. The pure space-based priming was not significant either, $F(1$, $21)=.14$. ns. The absence of pure object- and space-based priming might be due to lack of statistical power. Comparing to Experiment 1 using $44 \%$ of the data for evaluating pure object- or space-based priming, Experiment 2 used only $25 \%$ of the data for evaluating pure object- or space-based priming. ${ }^{1}$

To test the possibility of perceptual priming as being responsible for the current results, we conducted a within-subject analysis with two levels of orientation change condition (no-change if two successive trials had identical orientation and change if two successive trials had different orientations), two levels of current-trial validity (invalid-same and invalid-near) and two levels of previous object-cue validity (valid-object cue and invalid-object cue) as factors. If perceptual priming plays a role, there should be a larger priming effect when sequential trials have identical orientations. The results showed that there was no significant main effect of orientation change condition, $F(1,21)=1.46, p>.05$. Again it is most important to note that none of the interactions with the orientation change condition were significant, $F(1,21)<1$, ns; $F(1,21)<1$, ns; $F(1,21)<1$, ns; the interaction between orientation change and current-trial validity, orientation change and previous object-cue validity, and orientation change, currenttrial validity and previous object-cue validity, respectively.
We also tested if response priming might be contributing to the effects we observe by running a three-way ANOVA with two levels of response repetition condition (repeat if two successive trials had identical response and change if two successive trials had different responses), two levels of current-trial validity (invalidsame and invalid-near) and two levels of previous object-cue validity (valid-object cue and invalid-object cue) as factors. The result showed no interaction between response repetition, currenttrial validity and previous object-cue validity, $F(1,21)<1$, ns The object-based attentional effect is found only when the previous trial had a valid object cue, regardless of the response repetition condition, suggesting that the response repetition does not account the priming of object-based attention. ${ }^{2}$

The results of Experiment 2 match the results of Experiment 1. In both studies, the effectiveness of a cue in guiding attention to objects depended on whether the cue on the previous trial was a valid predictor of the object on which the target appears. Following a trial in which the cue and target appear on the same object, an object-based attentional effect is observed. In contrast, following a trial in which the cue and target appear on different objects, no evidence for the object-based allocation of attention is observed. Note that this result holds regardless of experiment-wise statistics: In Experiment 2, the cue provided no information about either the location of the target or the object in which the target would

\footnotetext{
${ }^{1}$ For similar reasons, we do not test pure object- and space-based priming effects in Experiment 3, in which only $4 \%$ of the data is used for testing pure space-based priming and $64 \%$ of the data for pure object-based priming, though a highly unbalanced distribution of trial types and resulted in many missing cells.

${ }^{2}$ We ran additional three-way ANOVAs with two levels of response repetition and pure object-based priming or pure space-based priming. The interaction between response repetition and pure object- or space-based priming was not significant, $F(1,20)=0.27, p=n s ; F(1,17)=0.60, p=$ $n s$. The three-way ANOVAs with repetition condition, current-trial validity, and previous object-cue condition were conducted only in Experiment 2. The response was not recorded in Experiment 1, and there were not enough observations to see the three-way interaction due to skewed probability in Experiment 3.
} 
appear, yet object-based attentional effects are observed when the previous trial suggested a dependency between cue and target.

A concern might be raised about Experiment 2 is that reducing the spatial validity of the cue $(25 \%)$ reduced both the space and object-based effects, which is seemingly inconsistent with the result by $\mathrm{He}$ et al. (2008) showing that reducing the spatial validity of the cue only decreased space-based attention. However, Experiment 2 has several procedural differences from He et al. First, the spatial cue validity used in the current study (25\%) is quite different from that used in $\mathrm{He}$ et al. In $\mathrm{He}$ et al., even the low validity condition had $50 \%$ spatial validity. Also the object validity of a cue was much higher in $\mathrm{He}$ et al. (76\% and $68 \%$ in high and low validity conditions, respectively) compared to Experiment 2 (50\%). Moreover, unlike $\mathrm{He}$ et al., Experiment 2 included the invalid-far condition. Because the target could appear in any display location, participants might have been encouraged to attend more broadly in our experiment, which might have slowed responses to targets following valid spatial or object cues (Davis et al., 2000).

\section{Experiment 3}

In Experiments 1 and 2, we showed a sequential effect involving control of object-based attention. Object-based attentional effects are observed on trial $n$ if and only if on trial $n-1$, the cue indicated the object on which a target would appear. We ruled out the possibility that this effect was due to repetition of perceptual features (e.g., the object shapes and locations) or repetition of responses, and thus the effect must be due to the abstract structural relationship between the cue and the object in which a target appears.

The sequential effects we observed in Experiments 1 and 2 appear to override cue-target contingencies that occur across the entire experiment. Over the course of Experiment 1, the cue validly predicted the object in which the target appeared on $66.67 \%$ of trials, yet a valid-object cue produced no facilitation when it followed a trial with an invalid-object cue. And although the object-cue validity dropped to $50 \%$ in Experiment 2, the object cueing effect seemed no different than in Experiment 1 when conditioned on the object-cue validity of the previous trial.

In Experiment 3, we conducted a further test of the hypothesis that long-term statistics of cue-target contingencies do not influence the effect we observe in our paradigm. We manipulated the relationship between cue and target such that the cue predicted the object on which the target would appear only $20 \%$ of trials, and consequently, a cue to one rectangle predicts that the target is likely to appear on the other rectangle. If control processes can suppress the operation of object-based attention when it is not useful globally, then local (sequential) object-based effects should be reduced or eliminated.

Shomstein and Yantis (2004) found evidence that object-based attention could be modulated by global statistics of the environment. They introduced spatial priors that operated in opposition to object-based cueing: On a large proportion of the invalid trials, the target appeared in a particular display location, which was orthogonal to the arrangement of objects. Consequently, directing attention to the cued object was far less useful than directing attention to the location with high prior probability. Shomstein and Yantis (2004) observed that participants were able to distribute attention spatially to exploit the spatial priors, and thereby mitigate the effects of object-based attention. However, this sensitivity to prior probabilities arose only at long SOAs $(600 \mathrm{~ms})$. At short SOAs (200, $400 \mathrm{~ms})$, the standard object-based cueing effect arose, suggesting that object-based attention is allocated rapidly and automatically, and responsiveness to global statistics has a slower time course. Our Experiments 1 and 2 used an SOA of $100 \mathrm{~ms}$. The Shomstein and Yantis (2004) study suggested that this SOA was too short to observe an effect of global statistics. Consequently, we included a longer cue-to-target SOA of $600 \mathrm{~ms}^{3}$ in the present experiment, as well as the brief $100 \mathrm{~ms}$ SOA.

\section{Method}

Participants. Forty participants (age range: 18-22 years) from the Urbana-Champaign community were recruited and paid to participate in a $1-\mathrm{hr}$ session. Twenty participants performed SOA $100 \mathrm{~ms}$ condition and 20 participants performed SOA $600 \mathrm{~ms}$ condition. They all had normal or corrected-to-normal visual acuity tested by Snellen Eye chart and normal color vision tested by Ishihara Test Chart Book for color deficiency. Participants signed an informed consent approved by the University of Illinois Institutional Review Board before they participated in the study.

Stimuli and procedure. Stimuli and procedure were identical to those in Experiment 2 with a few exceptions. First, in $70 \%$ of trials, a target was presented on the invalid-near condition. Valid, invalid-same and invalid-far conditions were presented on $10 \%$ of trials each. Second, the sequence of the orientation of display, the cue-target relation and target location were presented in random order. Finally, there were two cue-to-target SOA conditions (100 $\mathrm{ms}$ and $600 \mathrm{~ms}$ ). The SOA condition was a between-subject factor. Participants were not explicitly informed about the high probability of invalid-near condition before the experiment.

\section{Results and Discussion}

Figure 4 shows the RT results. Only trials in which responses to the target discrimination task were correct $(90 \%)$ were analyzed. RTs less than $150 \mathrm{~ms}$ and greater than $2,000 \mathrm{~ms}$ were not included in the analysis, and this trimming eliminated less than $1 \%$ of the data. Because the skewed probability of validity condition, there was relatively small number of observations on previous validobject cue condition. Therefore, a two-way ANOVA with previous object-cue validity and current-trial validity may provide a misleading view of the general pattern of validity effect. In Experiment 3, we conducted separate ANOVAs for current-trial validity and previous object-cue validity. The priming of object-based attention was examined by Wilcoxon signed-ranks test.

100-ms SOA. The ANOVA with current-trial validity as a within-subject factor demonstrated a significant validity effect, $F(2,38)=3.64, p<.05$. The valid trials showed the fastest RTs (531 ms), followed by invalid-same trials $(544.5 \mathrm{~ms})$, invalid-far (556 ms), and then invalid-near (561 ms). Pairwise comparisons with Bonferonni adjustment revealed a significant difference between the valid and invalid-near condition, $p<.05$. The differ-

\footnotetext{
${ }^{3}$ Thanks to an anonymous reviewer for suggesting we include the longer SOA condition.
} 


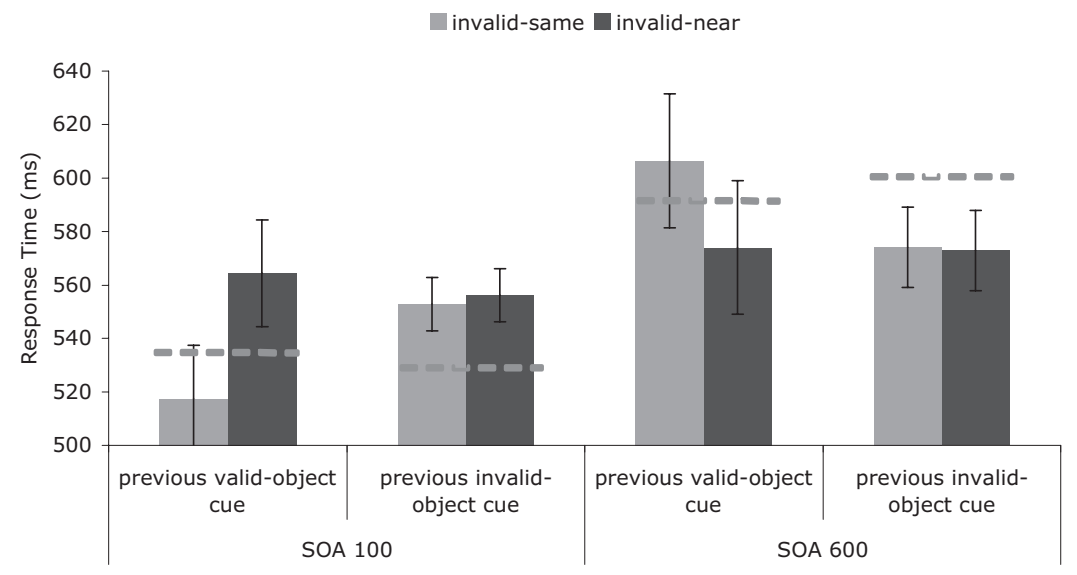

Figure 4. The results of Experiment 3 by previous object-cue validity and current-trial validity condition. Object-based attention effect was found only in the previous valid-object cue condition at short SOA. The horizontal dashed line represents the valid-cue condition. Error bars are within-subject $95 \%$ confidence intervals. SOA $=$ Stimulus-Onset Asynchrony.

ences between the valid and invalid-same and between the invalidsame and invalid-near conditions were not significant. The ANOVA with previous object-cue validity condition as withinsubject factors demonstrated no significant main effect, $F(1,19)<$ $1, n s$.

Next, we examined if the object-cue validity of the previous trial affects the deployment of object-based attention in the current trial using the Wilcoxon signed-ranks test. The object-cuing effect (i.e., faster RT for invalid-same than invalid-near) is significant only for the trials following the valid-object cue trial $(p<.01)$, not for trials following the invalid-object cue trial $(p=.57)$.

Accuracy data were analyzed in the same way as the RT data. The main effect of current-trial validity was not significant, $F(2$, $38)=1.08, p>.05$. The main effect of previous object-cue validity was significant, $F(1,19)=6.82, p<.05$. The previous invalid-object cue condition (90.8\%) showed higher accuracy than the previous valid-object cue condition (85.2\%). However, the main effect of previous object-cue validity might be because of skewed probability such that $80 \%$ of trials were previous invalidobject cue condition and $20 \%$ of trials were previous valid-object cue. Therefore responding inaccurately in the previous valid-object cue condition has a four fold larger effect than in the previous invalid-object cue condition.

600-ms SOA. The ANOVA with current-trial validity as a within-subject factor was not significant, $F(2,38)=1.83, p>.05$. The ANOVA with previous object-cue validity as within-subject factors demonstrated no significant main effect, $F(1,19)<1$, ns. Wilcoxon signed-ranks test showed that the object-based effect (i.e., faster RT for invalid-same than invalid-near) is not significant for both the trials following the valid-object cue trial $(p=.38)$, and for trials following the invalid-object cue trial $(p=.55)$.

Accuracy data were analyzed in the same way as the RT data. The main effect of current-trial validity was not significant, $F(2$, $38)<1, n s$. The main effect of previous object-cue condition was not significant, $F(1,19)<1$, ns.

Next, we performed an ANOVA with previous object-cue validity (valid-object cue, and invalid-object cue) and current-trial validity (invalid-same and invalid-near) as within-subject factors and SOA (100 ms and $600 \mathrm{~ms}$ ) as a between-subjects factor. The interaction between SOA, previous object-cue validity, and current-trial validity was significant, $F(1,38)=5.33, p<.05$. At the shorter SOA (100 ms), the object-based benefit was only significant on the previous valid-object cue condition. However at the longer SOA $(600 \mathrm{~ms})$, the object-based benefit was not significant on both previous valid- and invalid-object cue conditions.

In this experiment the target appears in the uncued object on $80 \%$ of the trials. If attentional control is adaptive to global statistics of the experiment, then object-based attention should not be allocated to the cued object. However, at the short cue-to-target SOA, an object-based cueing effect was observed, suggesting that control based on immediately recent experience is rapid and automatic. At the long cue-to-target SOA, no object-based cueing effect was observed. We attribute this finding to the operation of a secondary, slower, and perhaps voluntary mechanism that is sensitive to global statistics of the experiment. Our results and interpretation are consistent with those of Shomstein and Yantis (2004), who also distinguished between a rapid automatic mechanism and a slower strategy dependent on what the current behavioral context (e.g., global cue validity).

The results of Experiments 1 to 3 show a robust adaptation of attentional control to recent experience. At short SOAs, a cue will engage object-based attention if and only if on the previous trial the cue provided a valid indication of the object in which the target appeared. At long SOAs, as we showed in Experiment 3, this mechanism can be overridden by a secondary control strategy that utilizes statistics collected over a time window that extends beyond the immediately preceding trial, and probably reflects long-term learning about the task.

\section{General Discussion}

Our experiments demonstrate that recent experience performing a task can affect the engagement of object-based attention. In particular, the expression of object-based cueing in the Egly et al. (1994) task depends on whether the cue on the previous trial validly indicated the object on which a target would appear. The 
object-based cueing facilitation was found on trials following valid-object cue trials, but not on trials following invalid-object cue trials. Thus, object-based attention appears to be highly susceptible to trial-to-trial modulation. The Egly et al. task has given rise to a substantial literature on object-based attention, and it is likely that object-based cueing observed in this literature is attributable to sequential effects.

At short SOAs, the expression of object-based cueing does not depend on global cue validity (i.e., cue validity over a block of trials), suggesting that attentional control is responsive to immediately preceding trials, not long-term statistics of the environment. Even with the prior probability favoring the uncued object and no structure to the sequence of trials (Experiment 3), an object-based benefit is found on the trials following valid objectcue trials.

However, at longer SOAs, global statistics of the environment (i.e., probability manipulations) can override immediately preceding experience. We therefore conjecture, consistent with the claims of Shomstein and Yantis (2004), the existence of two distinct mechanisms involved in controlling the allocation of object-based attention: a rapid, automatic mechanism based on immediately preceding experience, and a slower, and perhaps voluntary, mechanism that is sensitive to global statistics of the environment.

Our work extends previous studies showing that control of attention is influenced by the ongoing stream of experience (see Kristjánsson, 2006, for a review). Many studies have explored priming of perceptual features such as color, orientation, shape, or location (Huang et al., 2004; Maljkovic \& Nakayama, 1994, 1996, 2000; Olivers \& Humphreys, 2003). Kristjánsson and colleagues (Kristjánsson et al., 2001; Kristjánsson \& Nakayama, 2003) showed that over one to four trials, abstract cue-target relationships can be learned, specifically the position of a target relative to features of the cue such as its location, color, or shape. Our research shows rapid (one trial) learning of an even more abstract relationship between cue and target-whether they are both associated with the same object.

It is important to note that our results are not due to repetition of perceptual features of displays. The location of the cue and target changed from trial to trial, as did the orientation of the rectangles that determined whether cue and target locations were part of the same object. In Experiment 2, the cue and target were presented with equal probability at each of the four ends of the rectangles. Therefore, the possibility of having two successive identical displays with identical cue-target location was very low. Moreover, when we compared performance on trials following an identical orientation display with trials following a different orientation display, there was no difference between those two conditions suggesting that the priming of the object-based attention is conceptual rather than perceptual. Finally, it is theoretically possible that the priming of object-based attention is a byproduct of response repetition. We analyzed the results of Experiment 2 by response repetition (true or false), current-trial validity (invalidsame and invalid-near) and previous object-cue validity to examine this possibility. We found no interaction between response repetition, current-trial validity, and previous object-cue validity suggesting that the priming of object-based attention was not affected by response repetition.

\section{Theories of Attentional Control}

Various theoretical perspectives have been proposed to explain the priming of pop out phenomenon. One suggestion is that the target-defining feature is primed on each trial, leading to greater attentional saliency for elements containing that same feature on the following trial, and quicker selection (Lee et al., 2009; Maljkovic \& Nakayama, 1994, 2000; Wolfe, Butcher, Lee, \& Hyle, 2003). Alternatively, each trial might produce an episodic memory representation, and when the current trial matches the memory of recent trials, selection and execution of a response is facilitated (Huang et al., 2004). Hybrid theories also have been proposed (Mozer, Shettel, \& Vecera, 2006). Although evidence has been presented in favor of the attentional-biasing account (Becker, 2008; Sigurdardottir et al., 2008), our experiments provide further evidence against a purely episodic-memory account based on the matches between a feature of recent trial and that of current trial in that we observe sequential dependencies between pairs of trials that have little in common in terms of the physical features of their stimuli.

Further, our experiments are consistent with the notion of attentional control settings, which specify modulations of the operation of visual attention in the service of performing a task or achieving a goal. Control settings have often been cast in terms of gains associated with each primitive feature dimension; these gains determine the ease with which features are able to grab attention. In the biased competition framework (Desimone \& Duncan, 1995), the gains can be implemented by pre-activating task-relevant features, which biases the outcome of the competition among features. In the guided search model (Wolfe, 1994, 2007), explicit top-down gain parameters are associated with each feature dimension, and the gain modulated feature activations sum to determine saliency.

Our experiments suggest the possibility of a control setting that determines the degree to which object-based attention should influence the prioritization of processing. What would such a control setting look like? If object-based attention is determined by grouping processes that select the set of locations associated with an object (Mozer, Zemel, Behrmann, \& Williams, 1992; Vecera, 1994; Vecera \& Farah, 1994; Weber, Kramer, \& Miller, 1997), then a control setting on object-based attention would modulate the strength of these grouping processes, or the ability of these grouping processes to influence a location-based saliency map.

Control settings are a means of using the same basic hardware to achieve flexible, task- and goal-relevant behavior. Control settings allow an individual to achieve optimal behavior in a given task and stimulus environment, subject to the constraints on the visual and attentional hardware (e.g., Mozer \& Baldwin, 2008; Mozer et al., 2006; Wolfe et al., 2003). From the perspective of optimality, object-based attention should be deployed in blocks of trials in which the cue tends to be a valid indicator of the target object (i.e., valid and invalid-same trials), but not when the cue provides no indication of the target object (i.e., invalid-different trials). Indeed, a type of inverse object-based attention should be deployed in conditions such as Experiment 3, where the cue is a reliable predictor of which object will not contain the target. At short SOAs, participants were clearly not optimal in this sense.

The priming of object-based attention observed in our experiments can be cast as adaptive behavior if individuals are operating 
in a highly dynamic, nonstationary environment, such that very recent experience is far more predictive of the future than more distant experience (Wilder, Jones, \& Mozer, 2009; Yu \& Cohen, 2008). In contrast, if individuals are operating in a static, stationary environment, recent experience is less reliable than regularities learned over a longer time scale. One might argue that naturalistic environments seldom require individuals to perform the same task repeatedly in exactly the same conditions, as is often required in blocked experimental trials, and thus, the assumption of nonstationarity seems a sensible default.

The implicit learning that takes place on a short time scale can be overridden when individuals have additional time to process a cue, as revealed in Experiment 3. This slower, secondary mechanism may involve explicit learning of regularities in the experimental task. Further research should examine the degree to which these two mechanisms are distinct and can be cast in terms of the contrast between implicit and explicit learning, or automatic and deliberate control. Together, these mechanisms point to the adaptive nature of attentional control that occurs on multiple time scales of experience.

\section{References}

Avrahami, J. (1999). Objects of attention, objects of perception. Perception \& Psychophysics, 61, 1604-1612. doi: 10.3758/BF03213121

Becker, S. I. (2008). The stage of priming: Are intertrial repetition effects attentional or decisional? Vision Research, 48, 664-684. doi: 10.1016/ j.visres.2007.10.025

Chen, Z., \& Cave, K. R. (2008). Object-based attention with endogenous cuing and positional certainty. Perception \& Psychophysics, 70, 14351443. doi: 10.3758/PP.70.8.1435

Chun, M. M., \& Jiang, Y. (1998). Contextual cueing: Implicit learning and memory of visual context guides spatial attention. Cognitive Psychology, 36, 28-71. doi: 10.1006/cogp.1998.0681

Chun, M. M., \& Nakayama, K. (2000). On the functional role of implicit visual memory for the adaptive deployment of attention across views. Visual Cognition, 7, 65-81. doi: 10.1080/135062800394685

Cosman, J. D., \& Vecera, S. P. (2010). Attentional capture under high perceptual load. Psychonomic Bulletin \& Review, 17, 815-820. doi: 10.3758/PBR.17.6.815

Davis, G., Driver, J., Pavani, F., \& Shepherd, A. (2000). Reappraising the apparent costs of attending to two separate visual objects. Vision Research, 40, 1323-1332. doi: 10.1016/S0042-6989(99)00189-3

Desimone, R., \& Duncan, J. (1995). Neural mechanisms of selective visual attention. Annual Review of Neuroscience, 18, 193-222. doi: 10.1146/ annurev.ne.18.030195.001205

Egly, R., Driver, J., \& Rafal, R. D. (1994). Shifting visual attention between objects and location: Evidence from normal and parietal lesion subjects. Journal of Experimental Psychology: General, 123, 161-177. doi: $10.1037 / 0096-3445.123 .2 .161$

Folk, C. L., \& Remington, R. W. (1998). Selectivity in attentional capture by featural singletons: Evidence for two forms of attentional capture. Journal of Experimental Psychology: Human Perception and Performance, 24, 847-858. doi: 10.1037/0096-1523.24.3.847

Folk, C. L., Remington, R. W., \& Johnson, J. C. (1992). Involuntary covert orienting is contingent on attentional control settings. Journal of Experimental Psychology: Human Perception and Performance, 18, 10301044. doi: 10.1037/0096-1523.18.4.1030

Folk, C. L., Remington, R. W., \& Wright, J. H. (1994). The structure of attentional control: Contingent attentional capture by apparent motion, abrupt onset, and color. Journal of Experimental Psychology: Human Perception and Performance, 20, 317-329. doi: 10.1037/00961523.20.2.317
Found, A., \& Muller, H. J. (1996). Searching for unknown feature targets on more than one dimention: Investigation a dimension-weighting account. Perception \& Psychophysics, 58, 88-101. doi: 10.3758/ BF03205479

Geyer, T., Müller, H. J., \& Krummenacher, J. (2008). Expectancies modulate attentional capture by salient color singletons. Vision Research, 48, 1315-1326. doi: 10.1016/j.visres.2008.02.006

He, X., Fan, S., Zhou, K., \& Chen, L. (2004). Cue validity and object-based attention. Journal of Cognitive Neuroscience, 16, 1085-1097. doi: $10.1162 / 0898929041502689$

Hecht, L. N., \& Vecera, S. P. (2007). Attentional selection of complex objects: Joint effects of surface uniformity and part structure. Psychonomic Bulletin \& Review, 14, 1205-1211. doi: 10.3758/BF03193114

Hillstrom, A. P. (2000). Repetition effects in visual search. Perception and Psychophysics, 62, 800-817. doi: 10.3758/BF03206924

Huang, L., Holcombe, A., \& Pashler, H. (2004). Repetition priming in visual search: Episodic retrieval, not feature priming. Memory \& Cognition, 32, 12-20. doi: 10.3758/BF03195816

Huang, L., \& Pashler, H. (2005). Quantifying object salience by equating distractor effects. Vision Research, 45, 1909-1920. doi: 10.1016/ j.visres.2005.01.013

Kramer, A. F., \& Jacobson, A. (1991). Perceptual organization and focused attention: The role of objects and proximity in visual processing. Perception and Psychophysics, 50, 267-284. doi: 10.3758/BF03206750

Kristjánsson, Á., \& Campana, G. (2010). Where perception meets memory: A review of repetition priming in visual search tasks. Attention, Perception and Psychophysics, 72, 5-18. doi: 10.3758/APP.72.1.5

Kristjánsson, Á. (2006). Rapid learning in attention shifts - A review. Visual Cognition, 13, 324-362.

Kristjánsson, Á., Mackeben, M., \& Nakayama, K. (2001). Rapid, objectbased learning in the deployment of transient attention. Perception, 30, 1375-1387. doi: 10.1068/p3251

Kristjánsson, Á., \& Nakayama, K. (2003). A primitive memory system for the deployment of transient attention. Perception and Psychophysics, 65, 711-724. doi: 10.3758/BF03194808

Lamy, D., Amunts, L., \& Bar-Haim, Y. (2008). Emotional priming of pop-out in visual search. Emotion, 8, 151-161. doi: 10.1037/15283542.8.2.151

Lamy, D., Yashar, A., \& Ruderman, L. (2010). A dual-stage account of inter-trial priming effects. Vision Research, 48, 1274-1279. doi: 10.1016/j.visres.2008.02.021

Lee, H., Mozer, M. C., \& Vecera, S. P. (2009). Mechanisms of priming of pop-out: Stored representations of feature-gain modulations. Attention, Perception and Psychophysics, 71, 1059-1071. doi: 10.3758/ APP.71.5.1059

Lee, H., \& Vecera, S. P. (2005). Visual cognition influences early vision: The role of visual short-term memory in amodal completion. Psychological Science, 16, 763-768. doi: 10.1111/j.1467-9280.2005.01611.x

Lee, H., \& Vecera, S. P. (2010). Spatial short-term memory assists in maintaining occluded objects. Psychonomic Bulletin \& Review. 17, 846-852. doi: 10.3758/PBR.17.6.846

Maljkovic, V., \& Nakayama, K. (1994). Priming of pop-out: I. Role of features. Memory \& Cognition, 22, 657-672. doi: 10.3758/BF03209251

Maljkovic, V., \& Nakayama, K. (1996). Priming of pop-out: II. Role of position. Perception and Psychophysics, 58, 977-991. doi: 10.3758/ BF03206826

Maljkovic, V., \& Nakayama, K. (2000). Priming of pop-out: III. A short term implicit memory system beneficial for rapid target selection. Visual Cognition, 7, 571-595. doi: 10.1080/135062800407202

McCarley, J. S., Kramer, A. F., \& Peterson, M. S. (2002). Overt and covert object-based attention. Psychonomic Bulletin and Review, 9, 751-758. doi: 10.3758/BF03196331

Meeter, M., \& Olivers, C. N. L. (2006). Intertrial priming stemming from 
ambiguity: A new account of priming in visual search. Visual Cognition, 13, 202-222. doi: 10.1080/13506280500277488

Moore, C., Yantis, S., \& Vaughan, B. (1998). Object-based visual selection: Evidence from perceptual completion. Psychological Science, 9, 104-110. doi: 10.1111/1467-9280.00019

Mortier, K., Theeuwes, J., \& Starreveld, P. (2005). Response selection modulates visual search within and across dimensions. Journal of Experimental Psychology: Human Perception and Performance, 31, 542557.

Mozer, M. C., \& Baldwin, D. S. (2008). Experience-guided search: A theory of attentional control. In J. Platt, D. Koller, \& Y. Singer (Eds.), Advances in neural information processing systems 20 (pp. 1033-1040). Cambridge, MA: MIT Press.

Mozer, M. C., Shettel, M., \& Vecera, S. P. (2006). Control of visual attention: A rational account. In Y. Weiss, B. Schoelkopf, \& J. Platt (Eds.), Advances in neural information processing systems 18 (pp. 923-930). Cambridge, MA: MIT Press.

Mozer, M. C., Zemel, R. S., Behrmann, M., \& Williams, C. K. I. (1992). Learning to segment images by dynamic feature binding. Neural Computation, 4, 650-665. doi: 10.1162/neco.1992.4.5.650

Müller, N. G., \& Kleinschmidt, A. (2003). Dynamic interaction of objectand space-based attention in retinotopic visual areas. Journal of Neuroscience, 23, 9812-9816.

Neo, G., \& Chua, F. K. (2006). Capturing focused attention. Attention, Perception \& Psychophysics, 68, 1286-1296.

Olivers, C. N. L., \& Humphreys, G. W. (2003). Visual marking inhibits singleton capture. Cognitive Psychology, 47, 1-42. doi: 10.1016/S00100285(03)00003-3

Pinto, Y., Olivers, C. N. L., \& Theeuwes, J. (2005). Target uncertainty does not lead to more distraction by singletons: Intertrial priming does. Perception \& Psychophysics, 67, 1354-1361. doi: 10.3758/BF03193640

Pratt, J., \& Sekuler, A. (2001). The effects of occlusion and past experience on the allocation of object-based attention. Psychonomic Bulletin \& Review, 8, 721-727. doi: 10.3758/BF03196209

Richard, A. M., Lee, H., \& Vecera, S. P. (2008). Attentional spreading in object-based attention. Journal of Experimental Psychology: Human Perception and Performance, 34, 842-853. doi: 10.1037/00961523.34.4.842

Shomstein, S., \& Yantis, S. (2002). Object-based attention: Sensory modulation or priority setting? Perception and Psychophysics, 64, 41-51. doi: 10.3758/BF03194556

Shomstein, S., \& Yantis, S. (2004). Configural and contextual prioritization in object-based attention. Psychonomic Bulletin \& Review, 11, 247-253. doi: 10.3758/BF03196566

Sigurdardottir, H. M., Kristjánsson, A., \& Driver, J. (2008). Repetition streaks increase perceptual sensitivity in brief visual search display. Visual Cognition, 16, 643-658. doi: 10.1080/13506280701218364

Soto, D., \& Blanco, M. J. (2004). Spatial attention and object-based attention: A comparison within a single task. Vision Research, 44, 69-81. doi: 10.1016/j.visres.2003.08.013
Töllner, T., Gramann, K., Müller, H. J., Kiss, M., \& Eimer, M. (2008). Electrophysiological markers of visual dimension changes and response changes. Journal of Experimental Psychology: Human Perception and Performance, 34, 531-542. doi: 10.1037/0096-1523.34.3.531

Töllner, T., Zehetleitner, M., Gramann, K., \& Müller, H. J. (2010). Topdown weighting of visual dimensions: Behavioral and electrophysiological evidence. Vision Research, 50, 1372-1381. doi: 10.1016/ j.visres.2009.11.009

Vecera, S. P. (1994). Grouped locations and object-based attention: Comment on Egly, Driver, \& Rafal (1994). Journal of Experimental Psychology: General, 123, 316-320. doi: 10.1037/0096-3445.123.3.316

Vecera, S. P., \& Farah, M. J. (1994). Does visual attention select objects or locations? Journal of Experimental Psychology: General, 123, 146-160. doi: 10.1037/0096-3445.123.2.146

Watson, S., \& Kramer, A. F. (1999). Object-based visual selective attention and perceptual organization. Perception and Psychophysics, 61, 31-49. doi: 10.3758/BF03211947

Weber, T., Kramer, A. F., \& Miller, G. (1997). Selective processing of superimposed objects: An electrophysiological analysis of object-based attentional selection. Biological Psychology, 45, 159-182. doi: 10.1016/ S0301-0511(96)05227-1

Wilder, M., Jones, M., \& Mozer, M. C. (2009). Sequential effects reflect parallel learning of multiple environmental regularities. In Y. Bengio, D. Schuurmans, J. Lafferty, C.K.I. Williams, \& A. Culotta (Eds.), Advances in Neural Information Processing Systems 22 (pp. 2053-2061). La Jolla, CA: NIPS Foundation.

Wolfe, J. M. (1994). Guided search 2.0: A revised model of visual search. Psychonomic Bulletin and Review, 1, 202-238. doi: 10.3758/ BF03200774

Wolfe, J. M. (2007). Guided search 4.0: Current progress with a model of visual search. In W. Gray (Ed.), Integrated models of cognitive systems (pp. 99-119). New York, NY: Oxford University Press.

Wolfe, J. M., Butcher, S. J., Lee, C., \& Hyle, M. (2003). Changing your mind: On the contributions of top-down and bottom-up guidance in visual search for feature singletons. Journal of Experimental Psychology: Human Perception and Performance, 29, 482-502. doi: 10.1037/ 0096-1523.29.2.483

Yu, A., \& Cohen, J. (2008). Sequential effects: Superstition or rational behavior? In D. Koller, D. Schuurmans, Y. Bengio, \& L. Bottou (Eds.), Advances in neural information processing systems 21 (pp. 1873-1880). Retrieved from http://books.nips.cc/papers/files/nips21/NIPS2008_0720.pdf

Zemel, R. S., Behrmann, M., Mozer, M. C., \& Bavelier, D. (2002) Experience-dependent perceptual grouping and object-based attention. Journal of Experimental Psychology: Human Perception and Performance, 28, 202-217. doi:10.1037/0096-1523.28.1.202

Received January 11, 2011

Revision received July 28, 2011

Accepted August 3, 2011 